\title{
Galilean covariance of quantum-classical hybrid systems of the Sudarshan type.
}

\author{
A. D. Bermúdez Manjarres \\ Universidad Distrital Francisco José de Caldas \\ Cra 7 No. 40B-53, Bogotá, Colombia \\ ad.bermudez168@uniandes.edu.co \\ N. Marín-Medina \\ Laboratorio de Investigación en Electronica y Redes- LIDER, Facultad de Ingeniería \\ Universidad Distrital Francisco José de Caldas \\ 110111, Bogotá, Colombia \\ nmarin@uniandes.edu.co
}

December 7, 2020

\begin{abstract}
We revisit quantum-classical hybrid systems of the Sudarshan type under the light of Galilean covariance. We show that these kind of hybrids cannot be given as a unitary representation of the Galilei group and at the same time conserve the total linear momentum unless the interaction term only depends on the relative canonical velocities.
\end{abstract}

\section{1 introduction}

Symmetry considerations play a fundamental role in modern physics, among other things, they serve as guiding principles for the development of new theories. It has been argued that there is a possibility that mesoscopic systems could be better described by a quantum-classical hybrid theory, whether it is a known theory or a yet-to-be discovered hybrid [1. Such theory might not be derivable as any limit of pure quantum mechanics. Given that both non-relativistic classical and quantum mechanics are Galilei covariant, it seems natural to impose Galilei covariance to any hybrid quantum-classical theory. In this work we investigate the consequences of Galilei covariance on an specific kind of hybrid systems, the sometimes called Sudarshan hybrids [2, 3, 4, 5.

Motivated by the measurement problem of quantum mechanics, Sudarshan and coworkers used the operational version of classical mechanics called the Koopman-von Neumann theory [6, 7] (hereafter abbreviated as the KvN theory) to couple a classical system with a quantum one. The KvN theory has 
the advantage of expressing classical mechanics in the same mathematical formalism used for quantum mechanics, i.e., the mathematics of operators acting on a Hilbert space. Sudarshan's hybrid theory was initially aimed to study the interaction of a quantum system with a classical measuring object. Peres and Terno objected the consistency of the hybrids of the Sudarshan type [8, 9]. Objections to general hybrid systems in more general contexts have been also stated [10, 11]. The above criticisms presuppose that the hybrid systems are a kind of partial classical limit of a fully quantum situation. Barceló et al [1] take the raised objections as an opportunity for the development of new theories in the mesoscopic level, theories that are not derivable from pure quantum mechanics.

It is natural to posit that any new theory in the non-relativistic regime should be Galilei covariant. The covariance of physical theories under groups of space-time transformation is the subject of too many publications to be listed here, we will only cite a handful of them. Non-relativistic quantum mechanics can be obtained as a irreducible unitary representation of the Galilei group [12, 13, 14, 15] while the structure of relativistic quantum field theories are determined by the properties of the Poincare group [16. On the classical part, non-relativistic classical mechanics has been derived from the structure of the Galilei group in the context of Lagrangian mechanics [12, 17] and as a canonical representation in terms of Poisson brackets in Hamiltonian mechanics [18, 19.

The differences in the approaches between the classical and the quantum representations of the Galilei group (unitary vs canonical representations) are due to the different mathematical formalism in which each theory is usually expressed. Nevertheless, the KvN theory and, hence, classical mechanics can also be obtained by considering an irreducible unitary representation of the Galilei group [20]. Thus, the Galilei covariance of the quantum and classical sectors of the Sudarshan Hybrids can both be studied within the context of unitary representations of Lie groups. The quantum and classical unitary representations of the Galilei group differ in several critical aspects that will be reviewed later in the main part of this work.

The main purpose of this work is to study the restrictions imposed by Galilei covariance on the hybrids of the Sudarshan type, whether they are understood as a partial limit of a quantum theory or a fully new theory on their own, .

This work is organized as follows. In section 2, a review of the KvN formalism is given; only the most necessary concepts are presented but references are given for a more in depth treatment of the theory.

In section 3 , we present a review of the quantum and the less-known classical unitary representation of the Galilei algebra. Again, only the necessary tools required for the study of Sudarshan hybrids are presented. For both cases only spinless particles are considered.

In section 4, the Galilei algebra for the hybrid system is constructed and the restriction imposed on the interaction Hamiltonian are studied. The main results of this work are derived here. The results can be summarized as follows:

1. Under very natural assumptions, the Galilei covariance of Sudarshan hy- 
brids restrict the choices of interaction terms between the quantum and classical systems. A list of all possible interaction terms is given.

2. None but one of the possible interaction terms conserve the total (quantum + classical) linear momentum.

3. Conservation of total momentum prevents any quantum back reaction on the classical observable variables.

The Einstein summation convention is used through all this work.

\section{The $\mathrm{KvN}$ formalism}

In the $\mathrm{KvN}$ operational formulation of classical mechanics of a point particle the position and momentum $(\hat{\mathbf{q}}, \hat{\mathbf{p}})$ are understood as Hermitian operators that commute with each other, and, therefore, no uncertainty principle is present between them. The theory also introduces two auxiliary Hermitian vector operators $\hat{\lambda}_{\mathbf{q}}$ and $\hat{\lambda}_{\mathbf{p}}$ that are understood to have no direct physical meaning and are deemed as unobservables. These four operators obey the following commutation relations

$$
\begin{aligned}
{\left[\hat{q}_{i}, \hat{q}_{j}\right] } & =\left[\hat{p}_{i}, \hat{p}_{j}\right]=\left[\hat{q}_{i}, \hat{p}_{j}\right]=\left[\hat{q}_{i}, \hat{\lambda}_{p_{j}}\right]=\left[\hat{p}_{i}, \hat{\lambda}_{q_{j}}\right]=0, \\
{\left[\hat{q}_{i}, \hat{\lambda}_{q_{j}}\right] } & =\left[\hat{p}_{i}, \hat{\lambda}_{p_{j}}\right]=i \delta_{i j} .
\end{aligned}
$$

The operators of the classical system act on a Hilbert space $\mathcal{H}_{c l}$ whose elements are vectors of the form $|\psi\rangle=\int\langle\mathbf{q}, \mathbf{p} \mid \psi\rangle|\mathbf{q}, \mathbf{p}\rangle d \mathbf{q} d \mathbf{p}$ such that the complex wavefunction $\psi(\mathbf{q}, \mathbf{p})=\langle\mathbf{q}, \mathbf{p} \mid \psi\rangle$ is square integrable. The wavefunction $\psi(\mathbf{q}, \mathbf{p})$ represents the probability amplitude of finding a particle in certain region of phase space, while the quantity $\rho=|\psi(\mathbf{q}, \mathbf{p})|^{2}$ is the probability density in phase space used in classical statistical mechanics. The kets $|\mathbf{q}, \mathbf{p}\rangle$ are eigenfunctions of $\hat{\mathbf{q}}$ and $\hat{\mathbf{p}}$

$$
\begin{aligned}
& \hat{q}_{i}|\mathbf{q}, \mathbf{p}\rangle=q_{i}|\mathbf{q}, \mathbf{p}\rangle, \\
& \hat{p}_{i}|\mathbf{q}, \mathbf{p}\rangle=p_{i}|\mathbf{q}, \mathbf{p}\rangle,
\end{aligned}
$$

and they obey the orthonnormality condition $\left\langle\mathbf{q}^{\prime}, \mathbf{p}^{\prime} \mid \mathbf{q}, \mathbf{p}\right\rangle=\delta\left(\mathbf{q}-\mathbf{q}^{\prime}\right) \delta\left(\mathbf{p}-\mathbf{p}^{\prime}\right)$.

If the classical system is governed by a Hamiltonian function $H_{c}(\mathbf{q}, \mathbf{p})$, then the evolution of $|\psi\rangle$ is ruled by a Schrödinger equation

$$
\frac{d}{d t}|\psi(t)\rangle=-i \hat{H}_{c l}|\psi(t)\rangle
$$

where $\hat{H}_{c l}$, known as the Liouvillian operator, is given by

$$
\hat{H}_{c l}=\nabla_{\mathbf{p}} H_{c} \cdot \hat{\lambda}_{\mathbf{q}}-\nabla_{\mathbf{q}} H_{c} \cdot \hat{\lambda}_{\mathbf{p}}
$$


For the typical classical Hamiltonian of a single particle $\left.H_{c}=\frac{\mathbf{p}^{2}}{2 m}+V(q)\right)^{1}$, the Liouvillian operator takes the form

$$
\hat{H}_{c l}=\frac{1}{m} \hat{\mathbf{p}} \cdot \hat{\lambda}_{\mathbf{q}}-\nabla_{\mathbf{q}} V(q) \cdot \hat{\lambda}_{\mathbf{p}}
$$

In the "wave mechanics" version of the theory, $\hat{\mathbf{q}}$ and $\hat{\mathbf{p}}$ act as multiplicative operators on $\psi(\mathbf{q}, \mathbf{p})$, while $\hat{\lambda}_{\mathbf{q}}$ and $\hat{\lambda}_{\mathbf{p}}$ act as derivatives

$$
\hat{\lambda}_{\mathbf{q}}=-i \nabla_{\mathbf{q}} ; \hat{\lambda}_{\mathbf{p}}=-i \nabla_{\mathbf{p}}
$$

In this version of the theory the Liouvillian becomes a differential operator whose action on phase space functions $\psi(\mathbf{q}, \mathbf{p})$ is given by

$$
\hat{H}_{c l} \psi=-i\left\{\psi, H_{c}\right\},
$$

where the above bracket is the Poisson bracket of Hamiltonian mechanics.

The Schrödinger equation (4) then reduces to

$$
\frac{\partial \psi}{\partial t}=-\left\{\psi, H_{c}\right\},
$$

Since $\hat{H}_{c l}$ is linear in $\hat{\lambda}_{\mathbf{q}}$ and $\hat{\lambda}_{\mathbf{p}}, \rho$ obeys the same equation as $\psi$

$$
\frac{\partial \rho}{\partial t}=-\left\{\rho, H_{c}\right\}
$$

Eq. (10) is the Liouville equation of classical statistical mechanics, and it is the proof that the abstract formulation in terms of operators of the KvN theory is equivalent to Hamiltonian mechanics.

Finally, let us mention that the operators of the set $\left\{\hat{\mathbf{q}}, \hat{\mathbf{p}}, \hat{\lambda}_{\mathbf{q}}, \hat{\lambda}_{\mathbf{p}}\right\}$ are selfandjoint on $\mathcal{H}_{c l}$, this set is also irreducible in this Hilbert space 2 .

\section{Review of the Quantum and the Classical rep- resentations of the Galilei Algebra}

The proper Galilei group is a ten parameter Lie group that consists of spacetime translations, rotations and transformations to moving frames (boosts). The group elements are to be realized by unitary transformation acting on the appropriated Hilbert space. To each space-time transformation there is associated an Hermitian operator, the generator of the transformation. The

\footnotetext{
${ }^{1}$ For simplicity, and since the main interest is in the classical-quantum coupling, we do not consider here the case where the classical system interact with an external force that have to be handled with the introduction of a vector potential.

${ }^{2}$ There is a possibility of adding internal degrees of freedom to the theory, $|\mathbf{q}, \mathbf{p}, \sigma\rangle$, so $\left\{\hat{\mathbf{q}}, \hat{\mathbf{p}}, \hat{\lambda}_{\mathbf{q}}, \hat{\lambda}_{\mathbf{p}}\right\}$ is not irreducible on $\mathcal{H}_{c l}$. In particular $\sigma$ may designate a classical spin for the classical point particle. The Galilei algebra allows for such classical spin, but here we only consider spinless particles.
} 
derivation of the Lie algebra associated to the Galilei group can be found, for example, in [14].

While both quantum and classical mechanics are associated to the same brackets relations, the realization of the Lie algebra generators is different in each case. In particular, the classical unitary representation of the Galilei algebra has only been recently considered [20] and it is less straightforward than the quantum representation (since the classical case necessarily involves nonobservable operators). For the above reasons, both representations shall be reviewed separately. Even if the reader is well versed in the Galilei group and algebra, it is recommended to follow closely the next two subsections as they fix the notation used later.

\subsection{Quantum Representation}

The position and momentum operator for the quantum system will be designated by $\hat{\mathbf{r}}$ and $\hat{\mathbf{k}}$, respectively. They, of course, obey the Heisenberg commutation relation $3\left[\hat{r}_{i}, \hat{k}_{j}\right]=i \delta_{i j}$. The generators of the Galilei algebra for the quantum sectors are: the translation operator, in the quantum case it coincides whit the momentum $\hat{\mathbf{k}}$; an operator for rotations $\hat{\mathbf{j}}$; the operator for the Galilean boosts $\hat{\mathbf{g}}$; the Hamiltonian $\hat{H}_{\mathrm{Q}}$ is the time translation operator; and the central charge of the algebra, $M$ (which will be considered here just as a real number). The Galilei algebra is a set of commutator equations that involves the generators of the space-time transformation, these equations are

$$
\begin{aligned}
& {\left[\hat{k}_{i}, \hat{k}_{j}\right]=\left[\hat{g}_{i}, \hat{g}_{j}\right]=\left[\hat{j}_{i}, \hat{H}_{\mathrm{Q}}\right]=\left[\hat{k}_{i}, \hat{H}_{\mathrm{Q}}\right]=0,} \\
& {\left[\hat{j}_{i}, \hat{j}_{j}\right]=i \varepsilon_{i j k} \hat{j}_{i} ;\left[j_{i}, \hat{k}_{j}\right]=i \varepsilon_{i j k} \hat{k}_{k} ;} \\
& {\left[\hat{j}_{i}, \hat{g}_{j}\right]=i \varepsilon_{i j k} \hat{g}_{k} ;\left[\hat{k}_{i}, \hat{g}_{j}\right]=i \delta_{i j} M ;\left[\hat{g}_{i}, \hat{H}_{\mathrm{Q}}\right]=i \hat{k}_{i} .}
\end{aligned}
$$

In the quantum case the generators of the algebra have well established physical meaning. $M$ is the mass (and for a physical representation $M$ is strictly a positive number), $\hat{\mathbf{k}}$ is the momentum, $\hat{\mathbf{j}}$ is the angular momentum, $\hat{H}_{\mathrm{Q}}$ is the energy (of a free particle) and $\hat{\mathrm{g}}$ is a physical quantity sometimes called the dynamic mass moment.

For a single spinless particle the momentum and position form an irreducible set of operators. The generators of the algebra are given in terms of $\hat{\mathbf{r}}$ and $\hat{\mathbf{k}}$ by

$$
\begin{aligned}
\hat{\mathbf{j}} & =\hat{\mathbf{r}} \times \hat{\mathbf{k}}, \\
\hat{\mathbf{g}} & =M \hat{\mathbf{r}}-t \hat{\mathbf{k}}, \\
\hat{H}_{\mathrm{Q}} & =\frac{\hat{k}^{2}}{2 M} .
\end{aligned}
$$

\footnotetext{
${ }^{3}$ Through all this work we set $\hbar=1$
} 
For systems of several particles the generators for individual particles can be combined to give generators for the entire systems. For example, for two particles the space translation are generated by the total linear momentum $\hat{\mathbf{k}}=\hat{\mathbf{k}}_{1}+\hat{\mathbf{k}}_{2}$ while rotations are generated by the total angular momentum $\hat{\mathbf{j}}=\hat{\mathbf{j}}_{1}+\hat{\mathbf{j}}_{2}$. The total Hamiltonian is allowed to have a extra term that account for the interaction between the two particles.

$$
\hat{H}_{\mathrm{Q}}=\frac{\hat{k}_{1}^{2}}{2 M_{1}}+\frac{\hat{k}_{2}^{2}}{2 M_{2}}+V .
$$

The Galilei algebra commutations relations not involving the Hamiltonian are all identically satisfied. The remaining relations give restrictions to the potential energy $V$. It can be shown that, in order for all the commutation equations to be satisfied, $V$ can only depend on scalar combinations of the relative position $\hat{\mathbf{r}}_{1}-\hat{\mathbf{r}}_{2}$ and the relative momentum $\hat{\mathbf{k}}_{1}-\hat{\mathbf{k}}_{2}$ [14]. It follows that the allowed total Hamiltonian is such that the total linear and angular momentum are constant of the motion.

\subsection{Classical Representation}

The KvN version of classical mechanics is most usually obtained starting from the Liouville equation, but it can also be derived as an irreducible unitary representation of the Galilei group where the operators of the algebra act on the Hilbert space $\mathcal{H}_{c l}$. To avoid confusion, when dealing with the classical representation we make the following change of notation for the operators of the Galilei algebra: $\hat{\mathcal{J}}$ is the operator of rotations, $\hat{\mathcal{G}}$ is associated to Galilean boosts, the operator $\hat{H}_{c l}$ gives time translation and $\hat{\lambda}_{\mathbf{q}}$ gives space translations. We maintain the symbol $M$ for the central charge appearing in the commutation relation for $\hat{\lambda}_{\mathbf{q}}$ and $\hat{\mathcal{G}}$

$$
\left[\hat{\lambda}_{q_{i}}, \hat{\mathcal{G}}_{j}\right]=i \delta_{i j} M
$$

In term of the irreducible set $\left\{\hat{\mathbf{q}}, \hat{\mathbf{p}}, \hat{\lambda}_{\mathbf{q}}, \hat{\lambda}_{\mathbf{p}}\right\}$ the elements of the Galilei algebra are given by

$$
\begin{aligned}
\hat{\mathcal{J}}_{i} & =\varepsilon_{i j k}\left(\hat{q}_{j} \hat{\lambda}_{q_{k}}+\hat{p}_{j} \hat{\lambda}_{p_{k}}\right), \\
\hat{\mathcal{G}}_{i} & =-\hat{\lambda}_{q_{i}} t-m \hat{\lambda}_{p_{i}}, \\
\hat{H}_{c l} & =\frac{1}{m} \hat{p}_{i} \hat{\lambda}_{q_{i}},
\end{aligned}
$$

where $m$ is a positive number interpreted as the mass. Let us stress here that the mass $m$ should not be confused with the central charge $M$. It can be checked by direct computation that operators (17) obey, with one notable exception, the same commutation relations as their quantum equivalents with the following replacement 


$$
\begin{aligned}
\hat{k}_{i} & \rightarrow \hat{\lambda}_{q_{i}}, \\
\hat{j}_{i} & \rightarrow \hat{\mathcal{J}}_{i}, \\
\hat{g}_{i} & \rightarrow \hat{\mathcal{G}}_{i}, \\
\hat{H}_{\mathrm{Q}} & \rightarrow \hat{H}_{c l} .
\end{aligned}
$$

In the "wave mechanics" version of the KvN theory, the Eqs.(7) and (8) explain how to get the Liouvillian from the classical Hamiltonian function via the Poisson bracket. The time translation operator appearing in (17) is obtained when the Hamiltonian of a free particle is used $H_{c}=\frac{\mathbf{p}^{2}}{2 m}$. The others generators can be written as Poisson brackets of phase space functions as follows $\hat{\lambda}_{\mathbf{q}}=$ $-i\{\cdot, \mathbf{p}\}, \hat{\mathcal{J}}=-i\{\cdot, \mathbf{L}\}$ and $\hat{\mathcal{G}}=-i\{\cdot, \mathbf{g}\}$, where $\mathbf{L}=\mathbf{r} \times \mathbf{p}$ is the angular momentum and $\mathbf{g}=m \mathbf{q}-t \mathbf{p}$ is the dynamic mass moment.

The only difference between the quantum and classical representation, albeit a remarkable one, comes from the central charge. In the quantum case $M$ is to be a positive number in order to have a physical representation of the group. However, in the classical case the only possible choice is $M=0$, so the central charge cannot be interpreted as the mass. In fact, the mass $m$ does not appear in the relations of in the Galilei algebra at all. The only different commutation relation, then, reads

$$
\left[\hat{\lambda}_{q_{i}}, \hat{\mathcal{G}}_{j}\right]=0
$$

Eq. (19) can be derived without any use of results from analytical dynamics [20], but it is also a direct consequence of the way the KvN theory assigns operators to phase space functions. It can be checked that the differential operators $\hat{\mathcal{J}}=$ $-i\{\cdot, \mathbf{L}\}$ and $\hat{\mathcal{G}}=-i\{\cdot, \mathbf{g}\}$ commute.

The above is highly striking when compared to the quantum case since quantums representation with vanishing $M$ are unphysical[12. However, this situation is not a problem since, unlike the quantum case, in the classical representation of the Galilei group the elements of the algebra have no direct physical meaning. For example, $\hat{\mathcal{J}}$ is an operator of rotations but not an angular momentum while $\hat{H}_{c l}$ generates time translation but its spectrum is not related to the energy of the system. By the same token, $M$ needs not to be related with the mass and it can vanish without causing any problem.

Just as in the quantum case, the Galilei algebra impose restrictions on the interaction between classical particles. It can be shown that the forces between the particles can only depend on scalar combinations of the relative position and the relative velocities, the conservation of the total linear momentum is closely related. The result just mentioned is known from analytical mechanics[19] but it can also be obtained from the classical unitary representation of the Galilei group in the context of the $\mathrm{KvN}$ theory [20. Let us take as an example a system of only two particles with velocity independent interaction. For this system the 
Liouvillian takes the form

$$
\hat{H}_{c l}=\frac{1}{m_{1}} \hat{\mathbf{p}}_{1} \cdot \hat{\lambda}_{\mathbf{q}_{1}}+\frac{1}{m_{2}} \hat{\mathbf{p}}_{2} \cdot \hat{\lambda}_{\mathbf{q}_{2}}-\nabla_{\mathbf{q}_{1}} V \cdot \hat{\lambda}_{\mathbf{p}_{1}}-\nabla_{\mathbf{q}_{2}} V \cdot \hat{\lambda}_{\mathbf{p}_{2}} \cdot
$$

The Eq.(20) can be obtained from symmetry principles though the procedure is somewhat convoluted [20. A second and simpler alternative consist in using the Poisson bracket formula (8) with the classical Hamiltonian given by $H_{c}=$ $\frac{\mathbf{p}_{1}^{2}}{2 m}+\frac{\mathbf{p}_{1}^{2}}{2 m}+V\left(\mathbf{q}_{1}-\mathbf{q}_{2}\right)$ as follows

$$
\begin{aligned}
\hat{H}_{c l} & =-i\left\{\cdot, H_{c}\right\}=\frac{-i}{m_{1}} \mathbf{p}_{1} \cdot \nabla_{\mathbf{q}_{1}}+\frac{-i}{m_{2}} \mathbf{p}_{2} \cdot \nabla_{\mathbf{q}_{2}} \\
& +i \nabla_{\mathbf{q}_{1}} V \cdot \nabla_{\mathbf{p}_{2}}+i \nabla_{\mathbf{q}_{2}} V \cdot \nabla_{\mathbf{p}_{2}} .
\end{aligned}
$$

The Eq.(20) is recovered by identifying the auxiliary operators $\hat{\lambda}$ in (21) with the use of the formulas given in (7). Since the potential depends on $\hat{\mathbf{q}}_{1}$ $\hat{\mathbf{q}}_{2}$, it follows that $\nabla_{\mathbf{q}_{1}} V=-\nabla_{\mathbf{q}_{2}} V$. The total linear momentum $\hat{\mathbf{p}}_{1}+\hat{\mathbf{p}}_{2}$ is a conserved quantity as it commutes with $\hat{H}_{c l}$. Let us notice that, while related, the conservation of the total momentum should not be confused with the conservation of the total translation operator $\hat{\lambda}_{\mathbf{q}_{1}}+\hat{\lambda}_{\mathbf{q}_{2}}$.

\section{Sudarshan Hybrids}

Having a formulation of classical mechanics in terms of operators acting on a Hilbert space, the quantum and classical sectors can be treated on the same footing. The Sudarshan hybridization consist in coupling the quantum and classical systems using a tensor product, just as to pure quantum systems are coupled in the standard quantum theory. The space of states for the hybrid system is $\mathcal{H}_{T}=\mathcal{H}_{\mathrm{Q}} \otimes \mathcal{H}_{c l}$. It follows from its definition that in the joint system the quantum operators commute with the classical ones.

Just as in pure quantum mechanics, the generators of space-time transformation with purely geometrical interpretation (space translation, rotations and boosts) acting on the joint space $\mathcal{H}_{T}$ are made by adding the corresponding operators for the individual systems

$$
\begin{aligned}
\hat{\mathscr{P}}_{i} & =\hat{k}_{i}+\hat{\lambda}_{q_{i}}, \\
\hat{G}_{i} & =\hat{g}_{i}+\hat{\mathcal{G}}_{i}, \\
\hat{\mathscr{J}}_{i} & =\hat{j}_{i}+\hat{\mathcal{J}}_{i} .
\end{aligned}
$$

It is worth noting that while $\hat{\mathscr{P}}$ and $\hat{\mathscr{J}}$ generate translations and rotations for the joint system, they are not the total linear momentum nor the total angular momentum. When the systems are non-interacting, the total momentum is given by $\hat{\mathbf{P}}_{t}=\hat{\mathbf{k}}+\hat{\mathbf{p}}$, and it is a constant of the motion. It seems most natural to keep $\hat{\mathbf{P}}_{t}$ as the total momentum when the interaction is switched on. 
The generator of time translation is allowed to have an extra term that accounts for the interaction of the two systems

$$
\hat{H}_{T}=\hat{H}_{\mathrm{Q}}+\hat{H}_{c l}+\hat{H}_{i n t} .
$$

All commutators of the Galilei algebra not involving $\hat{H}_{T}$ are identically satisfied. In particular, the mass of the quantum system (and not the total mass) becomes again the central charge of the coupled algebra

$$
\left[\hat{\mathscr{P}}_{i}, \hat{G}_{i}\right]=\delta_{i j} M \text {. }
$$

On the other hand, the commutators involving $\hat{H}_{T}$ restrict the possible choices for $\hat{H}_{\text {int }}$. From the commutation relations

$$
\left[\hat{\mathscr{J}}_{i}, \hat{H}_{\mathrm{T}}\right]=\left[\hat{\mathscr{P}}_{i}, \hat{H}_{\mathrm{T}}\right]=0 ;\left[\hat{G}_{i}, \hat{H}_{T}\right]=i \hat{\mathscr{P}}_{i},
$$

it follows that the interaction term has to obey the following conditions

$$
\begin{aligned}
{\left[\hat{\mathscr{P}}_{i}, \hat{H}_{\text {int }}\right] } & =0, \\
{\left[\hat{G}_{i}, \hat{H}_{\text {int }}\right] } & =0, \\
{\left[\hat{\mathscr{J}}_{i}, \hat{H}_{\text {int }}\right] } & =0 .
\end{aligned}
$$

Before studying the restriction imposed on $\hat{H}_{\text {int }}$ by the above equations, let us discuss a further requirements that comes solely from the classical sector. From the expression given for $H_{c l}$ of a free particle (17) and the equation $\hat{\mathbf{p}}=$ $i m\left[\hat{\mathbf{q}}, \hat{H}_{T}\right]$, it is obtained that $\left[\hat{\mathbf{q}}, \hat{H}_{i n t}\right]=0$. The above means the interaction term is independent of $\hat{\lambda}_{\mathbf{q}}$. On the other hand, the acceleration operator for the classical sector is related to the classical momentum by

$$
\hat{\mathbf{a}}=\frac{i}{m}\left[\hat{\mathbf{p}}, \hat{H}_{T}\right]=\frac{i}{m}\left[\hat{\mathbf{p}}, \hat{H}_{i n t}\right] .
$$

If the acceleration is to be an observable, then it cannot depend on the nonobservable operators $\hat{\lambda}_{\mathbf{q}}$ and $\hat{\lambda}_{\mathbf{p}}$. Hence, from (26), we find again that $\hat{H}_{\text {int }}$ has to be independent on $\hat{\lambda}_{\mathbf{q}}$, and, additionally, $\hat{H}_{\text {int }}$ has to be at most linear on $\hat{\lambda}_{\mathbf{p}}$.

Now, returning to the conditions from the Galilei algebra, we have from Eq. (25a) that $\hat{H}_{\text {int }}$ can only be function of the relative position $\hat{\mathbf{r}}-\hat{\mathbf{q}}$ since other combinations of $\hat{\mathbf{r}}$ and $\hat{\mathbf{q}}$ do not commute with $\hat{\mathscr{P}}_{i}$. By the same token, Eq. (25b) implies that $\hat{H}_{\text {int }}$ can only be function of the relative velocity $\frac{\hat{\mathbf{k}}}{M}-\frac{\hat{\mathbf{p}}}{m}$. No equation from the algebra restrict the dependence of $\hat{H}_{\text {int }}$ on $\hat{\lambda}_{\mathrm{p}}$. Finally, Eq. (25c) can only be satisfied if $\hat{H}_{i n t}$ is an scalar operator. Hence, the interaction term can only be constructed from the following scalar combinations: $(\hat{\mathbf{r}}-\hat{\mathbf{q}})^{2}$, $\left(\frac{\hat{\mathbf{k}}}{M}-\frac{\hat{\mathbf{p}}}{m}\right)^{2},(\hat{\mathbf{r}}-\hat{\mathbf{q}}) \cdot\left(\frac{\hat{\mathbf{k}}}{M}-\frac{\hat{\mathbf{p}}}{m}\right),(\hat{\mathbf{r}}-\hat{\mathbf{q}}) \cdot \hat{\lambda}_{\mathbf{p}}$ and $\left(\frac{\hat{\mathbf{k}}}{M}-\frac{\hat{\mathbf{p}}}{m}\right) \cdot \hat{\lambda}_{\mathbf{p}}$. 
Of the above, only $\left(\frac{\hat{\mathbf{k}}}{M}-\frac{\hat{\mathbf{p}}}{m}\right)^{2}$ commutes with the total linear momentum $\hat{\mathbf{k}}+\hat{\mathbf{p}}$. Thus, in a Sudarshan hybrid only interactions that depend on the relative canonical velocity conserve the total momentum. Moreover, only momentum non-conserving interaction can have a quantum back reaction on the classical observable variables since both $\hat{\mathbf{q}}$ and $\hat{\mathbf{p}}$ commute with $\left(\frac{\hat{\mathbf{k}}}{M}-\frac{\hat{\mathbf{p}}}{m}\right)^{2}$.

\section{Discussion and Final Comments}

The present work does not tell whether a quantum-classical hybrid theory is a good way to describe aspects of the real world or not. What has been done here is to find what conditions a Sudarshan hybrid must fulfill in order to be Galilei covariant.

It is worth noting that in the literature there can be found interaction terms that do not respect the commutation relations from the Galilei algebra for the total system (see, for example, equation (13) in Ref [1] or equation (18) in Ref [8]. See also [21]). It was noted in [8] that a quantum-classical coupling need not to conserve the total energy, here a more general result was given since conditions for the conservation of the total momentum were obtained.

The non-conservation of the total momentum for most of the allowed interaction terms is a strange result. Galieli covariance guarantee conservation of momentum in both quantum and classical mechanics but the same Lie algebra has almost the opposite result for Sudarshan hybrids.

Restricting the theory so only interaction depending on the relative velocity are considered is very harsh and seems to compromise any actual application to real situations. On the other hand, allowing position-dependent interactions requires the abandonment of a well tested conservation law in both the classical and quantum regime. It is true that there are new conserved quantities, like the total translation operator $\hat{\mathscr{P}}=\hat{\mathbf{k}}+\hat{\lambda}_{\mathbf{q}}$. However, the possible physical meaning of $\hat{\mathscr{P}}$ is not clear as it depends on the classically unobservable $\hat{\lambda}_{\mathbf{q}}$. The

results derived in this work seem to put in serious doubt the physical viability of Sudarshan hybrids as independent (non-quantum derivable) theories.

\section{References}

[1] C. Barcel Barceló, R. Carballo-Rubio, L.J Garay and R. Gómez-Escalante, Phys. Rev. A 86, 042120 (2012).

[2] E. C. G. Sudarshan, Pramana 6, 117 (1976).

[3] T. N. Sherry and E. C. G. Sudarshan, Phys. Rev. D 18, 4580 (1978).

[4] T. N. Sherry and E. C. G. Sudarshan, Phys. Rev. D 20, 857 (1979).

[5] S. R. Gautam, T. N. Sherry, and E. C. G. Sudarshan, Phys. Rev. D 20, 3081 (1979). 
[6] B. O. Koopman, Proc. Natl. Acad. Sci. U.S.A. 17, 315 (1931).

[7] J. von Neumann, Ann. Math. 33, 587 (1932).

[8] A. Peres and D. R. Terno, Phys. Rev. A 63, 022101 (2001).

[9] D. R. Terno, Found. Phys. 36, 102 (2006).

[10] L. L. Salcedo, Phys. Rev. A54, 3657 (1996).

[11] L. L. Salcedo, Phys. Rev. A85, 022127 (2012).

[12] J. -M. Lévy-Leblond, "Galilei group and Galilean invariance, Group Theory and its Applications", Vol. 2, ( Academic press: New York ; 1971).

[13] J. M. Jauch, " Foundation of Quantum Mechanics", ( Addison-Wesley Publishing Co, Reading, Massachussetts; 1968).

[14] T. F. Jordan, "Linear Operators for Quantum Mechanics", (John Wiley \& Sons, New York; 1969).

[15] L. E. Ballentine, "Quantum Mechanics: A Modern Development", (World Scientific, Singapore; 1998).

[16] S. Weinberg, "The Quantum Theory of Fields", Vol 1, (Cambridge University Press, Cambridge, 1995).

[17] L. Landau and E. M. Lifshitz, "Mechanics", (Pergamon, New York, 1958).

[18] M. Pauri and G. Prosperi, J. Math. Phys. 9, 1146 (1968) .

[19] E.C.G. Sudarshan and N. Mukunda, "Classical Dynamics : A Modern Perspective", (Wiley, New York, 1974).

[20] A. D. Bermúdez Manjarres, M. Nowakowski and D. Batic, Ann. Phys. (NY) 416, $168157(2020)$

[21] M. Radonjić, D. B. Popović, S. Prvanović, and N. Burić, Phys. Rev. A 89, 024104 (2014). 